\title{
Modulation-based time-variant filter design using Wigner distribution
}

\author{
Soo-Chang Pei*, Min-Hung Yeh \\ Department of Electrical Engineering, National Taiwan University, Taipei, Taiwan. 10764, ROC
}

Received 22 July 1993; revised 20 December 1993, 11 February 1994 and 5 May 1994

\begin{abstract}
The modulation-based time-variant filters are very useful for nonstationary signal processing. They can be constructed by three stages: pre-modulation, time-invariant low-pass filtering and post-modulation. Wigner distribution is a popular tool for showing the time-frequency distribution of nonstationary signals. The combination of these two techniques is presented in this paper. We introduce Wigner distribution as a tool to estimate the modulation function for modulation-based filter. The Wigner distributions of modulation-based filters are discussed and the Wigner distributions of output signals of modulation-based filters are also shown.
\end{abstract}

\section{Zusammenfassung}

Modulationsbasierte zeitvariante Filter sind sehr nützlich zur Verarbeitung nichtstationärer Signale. Sie können in drei Stufen konstruiert werden: Vormodulation, zeitinvariante Tiefpaß-Filterung und Nachmodulation. Zur Darstellung der Zeit-Frequenz-Verteilung ist die Wigner-Verteilung ein verbreitetes Hilfsmittel. Eine Kombination dieser beiden Techniken wird in dieser Arbeit dargestellt. Wir führen die Wigner-Verteilung als Methode zur Schätzung der Modulationsfunktion für ein modulationsbasiertes Filter ein. Die Wigner-Verteilungen von modulationsbasierten Filtern werden diskutiert und die Wigner-Verteilungen der Ausgangssignale von modulationsbasierten Filtern gezeigt.

\section{Résumé}

Les filtres basés sur la modulation et variant temporellement sont très utiles pour le traitement des signaux non-stationnaires. Ils peuvent être construit en trois étapes: la prémodulation, le filtrage passe-bas invariant dans le temps et la post-modulation. La distribution de Wigner est un outils populaire pour montrer la distribution temps-fréquence des signaux non-stationnaires. La combinaison de ces deux techniques est présentée dans cet article. Nous introduisons la distribution de Wigner comme un outils pour l'estimation de la fonction de modulation pour les filtres basés sur la modulation. Les distributions de Wigner des filtres basés sur la modulation sont discutées et les distributions de Wigner des signaux de sortie des filtres basés sur la modulation sont également montrées.

Keywords: Time-variant filter; Wigner distribution

\footnotetext{
* Corresponding author.
} 


\section{Introduction}

Conventional time-invariant FIR and IIR filters are designed to extract the desired component from received signals. These filters are only suitable for stationary signal processing. While we deal with a nonstationary signal, time-variant filters must be used. The theory in the LTI (linear time-invariant) filters has been well developed [6]. Huang and Aggarwal [3] have extended the theory in LTI filters to analyze the LTV (linear time-variant) filters. One simple method to design time-variant filters is to combine modulation technique and time-invariant filters. These filters are constructed by three stages: pre-modulation, low-pass filtering and post-modulation. They are called modulationbased filters. Furthermore, Wigner distribution (WD) is a very popular tool to observe the time-frequency distribution of nonstationary signals [2]. The combination of these two techniques is presented in this paper. We introduce Wigner distribution as a tool to estimate the modulation function for modulation-based filter. Wigner distributions of modulation-based filters are discussed and the Wigner distributions of output signals for modulation-based filters are also shown.

\section{Wigner distributions of modulation-based time-variant filters}

\subsection{Wigner relations between LTI filters and LTV filters}

For convenience of further discussion, the relations between LTI filters and LTV filters are reviewed. The LTI filter is only a special case of LTV filter, thus can be regarded as an LTV filter. The corresponding LTV impulse response can be obtained from the original LTI impulse response $h_{i}(t)$ by

$h_{v}\left(t, t^{\prime}\right)=h_{i}\left(t-t^{\prime}\right)$.

Wigner distribution is a popular tool for nonstationary signal analysis. The Wigner distribution of signal $x(t)$ is defined as [2]

$$
W_{x}(t, \omega)=\int_{-\infty}^{\infty} x(t+\tau / 2) x^{*}(t-\tau / 2) \mathrm{e}^{-\mathrm{j} \omega \tau} \mathrm{d} \tau .
$$

The 2-D Wigner distribution of 2-D signal $f(x, y)$ is defined as

$$
\begin{aligned}
W_{f}(x, y, u, v) & =\int_{-\infty}^{\infty} \int_{-\infty}^{\infty} f(x+\alpha / 2, y+\beta / 2) \\
& \times f^{*}(x-\alpha / 2, y-\beta / 2) \mathrm{e}^{-\mathrm{j}(\alpha u+\beta v)} \mathrm{d} \alpha \mathrm{d} \beta .
\end{aligned}
$$

Several properties of 2-D Wigner distribution are listed in [4] and are beneficial to further development of this paper.

In LTI filter, the Wigner distribution of output signals can be obtained by the time-convolution between the Wigner distribution of input signal and that of filter impulse response [2].

$$
\begin{aligned}
W_{y}(t, \omega) & =\int_{-\infty}^{\infty} W_{h_{i}}(t-\hat{t}, \omega) W_{x}(\hat{t}, \omega) \mathrm{d} \hat{t} \\
& =W_{h_{i}}(t, \omega) \star_{t} W_{x}(t, \omega),
\end{aligned}
$$

where $W_{h_{i}}(t, \omega)$ is the Wigner distribution of $h_{i}(t)$, $\star_{t}$ is the convolution operator in variable $t$.

In LTV filter, this relation becomes [7]

$$
\begin{aligned}
& W_{y}(t, \omega) \\
& \quad=\frac{1}{2 \pi} \int_{-\infty}^{\infty} \int_{-\infty}^{\infty} W_{h_{v}}\left(t, t^{\prime}, \omega,-\omega^{\prime}\right) W_{x}\left(t^{\prime}, \omega^{\prime}\right) \mathrm{d} t^{\prime} \mathrm{d} \omega^{\prime},
\end{aligned}
$$

where $W_{h_{v}}\left(t, t^{\prime}, \omega, \omega^{\prime}\right)$ is the 2-D Wigner distribution of $h_{v}\left(t, t^{\prime}\right)$. Thus, the Wigner distribution of the output signal can be calculated by Eq. (6). When the LTI filter is treated as an LTV filter, its Wigner distribution becomes

$W_{h_{v}}\left(t, t^{\prime}, \omega,-\omega^{\prime}\right)=2 \pi \delta\left(\omega-\omega^{\prime}\right) W_{h_{i}}\left(t-t^{\prime}, \omega\right)$,

where $\delta(\cdot)$ is delta function. The proof of Eq. (7) is listed in Appendix A.

\subsection{Modulation-based LTV filter}

The basic idea of modulation-based filters is to try passing time-variant signal band in time-frequency plane. Based upon this notation, time-variant filter can be implemented by a three-stage processe: pre-modulation, LTI filtering, and postmodulation. Fig. 1 lists the block diagram of modulation-based filter. $m(t)=\mathrm{e}^{\mathrm{j} \phi(t)}$ is called modulation 


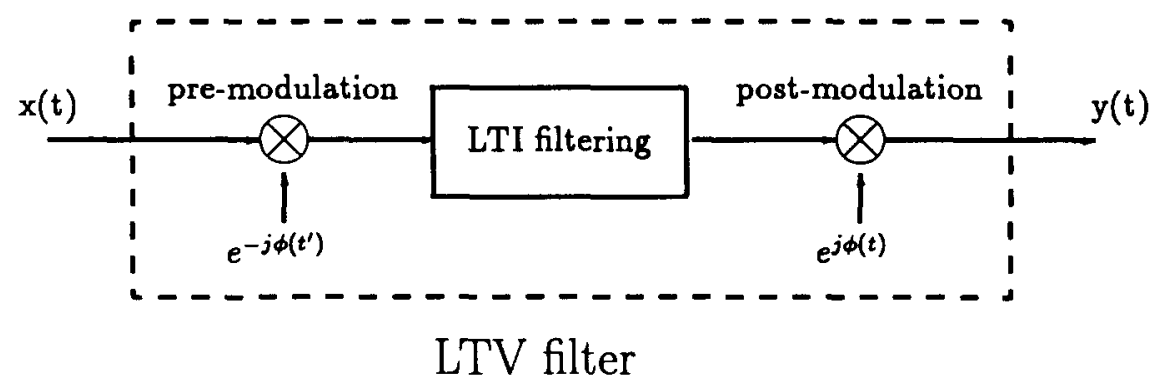

Fig. 1. The three stages of modulation-based filter.

function. The derivative of $\phi(t)$ is the center frequency of the desired time-varying band. It can be estimated by phase-locked loop [5] or Wigner distribution of received signal. The phases in pre-modulation and post-modulation are opposite signed. Because the processing of LTI filtering will cause some delay, there must be some delay in the phase of post-modulation comparing to that of pre-modulation. This delay depends upon the filter length in the LTI filter. Even if the output $y(t)$ is a complex signal, the real part can be taken to obtain what we wanted. The LTI filter in Fig. 1 is an FIR low-pass filter. The relation between input signals and output signals is

$$
\begin{aligned}
y(t) & =\int_{-\infty}^{\infty} \mathrm{e}^{-\mathrm{j} \phi\left(t^{\prime}\right)} h_{i}\left(t-t^{\prime}\right) \mathrm{e}^{\mathrm{j} \phi(t)} x\left(t^{\prime}\right) \mathrm{d} t^{\prime} \\
& =\int_{-\infty}^{\infty} h_{v}\left(t, t^{\prime}\right) x\left(t^{\prime}\right) \mathrm{d} t^{\prime},
\end{aligned}
$$

where $h_{v}\left(t, t^{\prime}\right)=h_{i}\left(t-t^{\prime}\right) \mathrm{e}^{\mathrm{j}\left(\phi(t)-\phi\left(t^{\prime}\right)\right)}$ is the impulse response of convolution-based time-variant filter.

\subsection{Wigner distributions of modulation-based filters}

By Eq. (6), we can get the Wigner distribution of the output signal from the Wigner distribution of input signal and LTV transfer function. Moreover, let us consider the Wigner distributions of modulation-based filters. The Wigner distributions of these filters are

$$
\begin{aligned}
W_{h_{\nu}}\left(t, t^{\prime}, \omega,-\omega^{\prime}\right)= & 2 \pi \delta\left(\omega-\omega^{\prime}\right) W_{h_{t}}\left(t-t^{\prime}, \omega\right) \\
& \star_{\omega} W_{m}(t, \omega) \star_{\omega^{\prime}} W_{m}\left(t^{\prime}, \omega^{\prime}\right),
\end{aligned}
$$

where $W_{m}(t, \omega)$ is the Wigner distribution of modulation function $m(t)=\mathrm{e}^{\mathrm{j} \phi(t)}, \star_{\omega}$ denotes the convolution operator in $\omega$. The proof of Eq. (10) is also listed in Appendix A. Using Eq. (10), the Wigner distribution of modulation-based filter can be obtained from the Wigner distribution of modulation function and FIR filter.

Now, we will discuss the behavior of special modulating function $m(t)=\mathrm{e}^{\mathrm{j} \phi(t)}$.

(1) Constant modulation $(\phi(t)=k t)$ : In this case there are no approximations in the transfer function. Because $H_{v}(t, \omega)=H_{i}(\omega-k)$, an LTI bandpass filter can be obtained. The Wigner distribution of this filter is

$$
\begin{aligned}
& W_{h_{\nu}}\left(t, t^{\prime}, \omega,-\omega^{\prime}\right) \\
& \quad=2 \pi\left[\delta\left(\omega-\omega^{\prime}\right) W_{h_{i}}\left(t-t^{\prime}, \omega-k\right)\right] .
\end{aligned}
$$

(2) Linear $F M\left(\phi(t)=\frac{1}{2} a t^{2}+b t\right)$ : In this case the modulation function is treated as a chirp signal. $W_{m}(t, \omega)=\delta(\omega-a t-b)$. We can get a closed form for the Wigner distribution of modulation-based filter.

$$
\begin{aligned}
W_{h_{v}}\left(t, t^{\prime}, \omega,-\omega^{\prime}\right)= & 2 \pi \delta\left[\omega-\omega^{\prime}-a\left(t-t^{\prime}\right)\right] \\
& \times W_{h_{i}}\left(t-t^{\prime}, \omega-a t-b\right) .
\end{aligned}
$$

(3) Quadratic $F M\left(\phi(t)=\frac{1}{3} a t^{3}+\frac{1}{2} b t^{2}+c t\right)$ : If the degree of $\phi(t)$ is greater than 2 , there is no closed form in Wigner distribution for this modulation function. So there is also no closed form for modulation-based filter. 

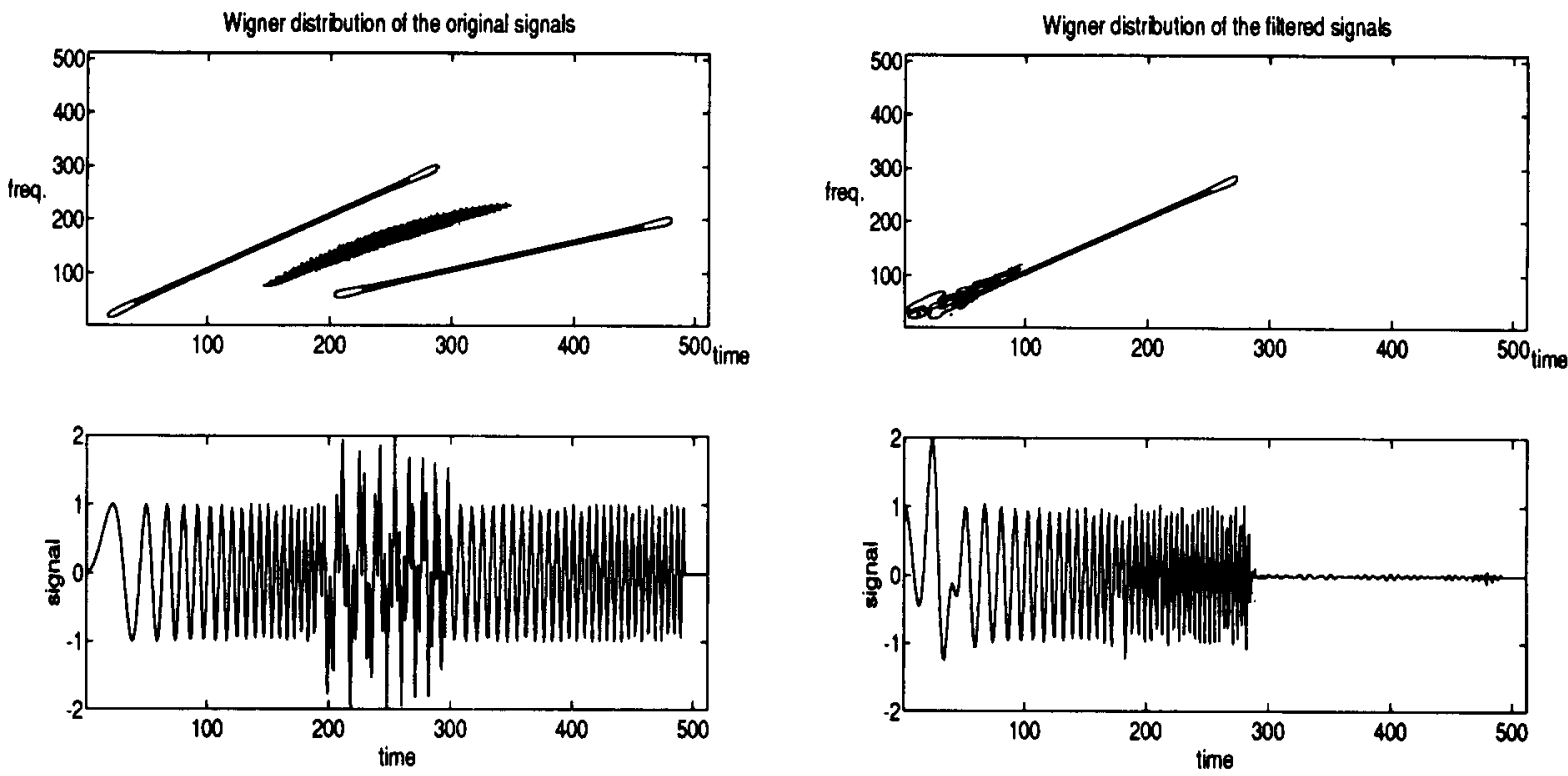

Fig. 2. The original and their filtered signals in Example 1.
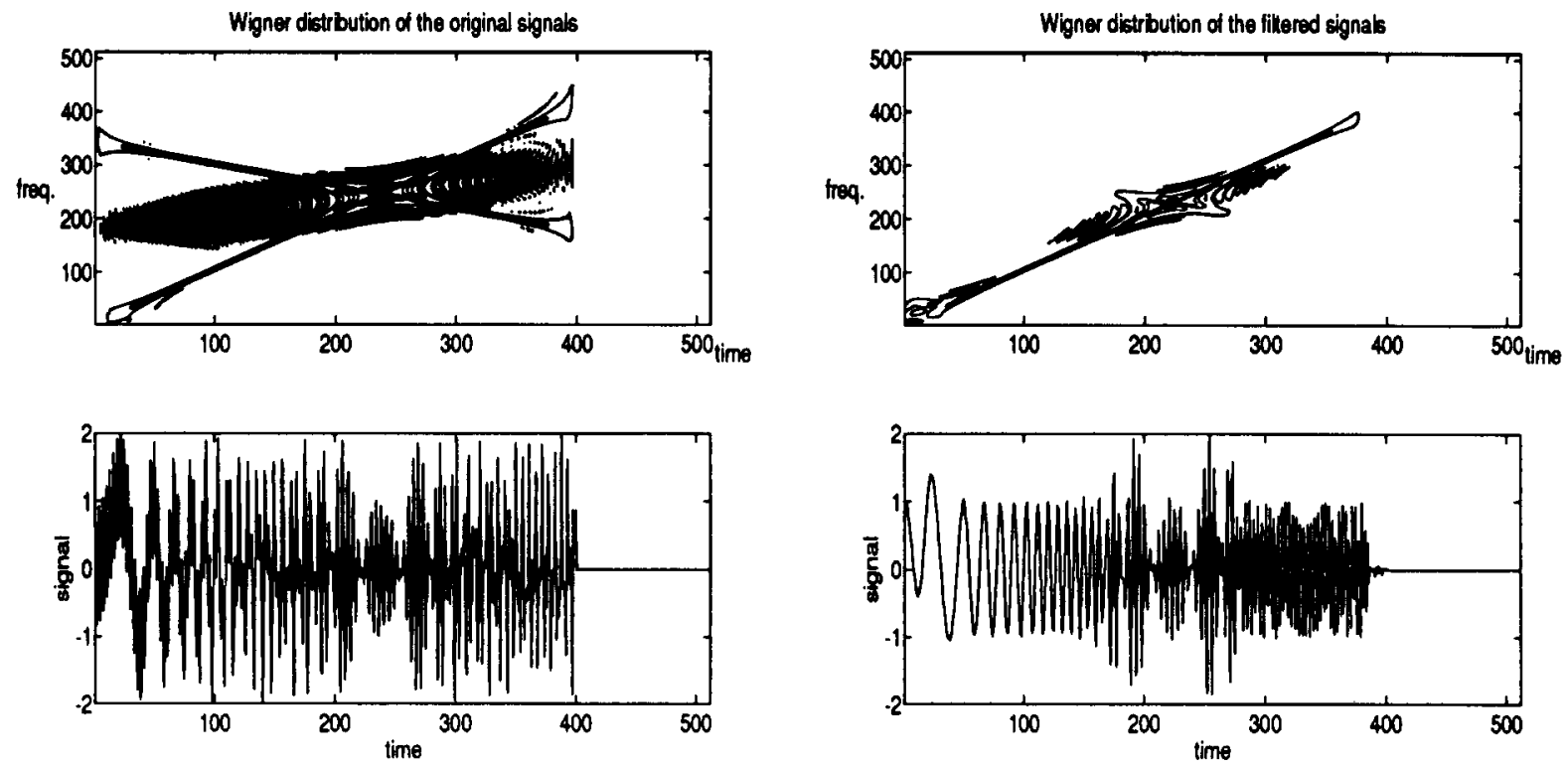

Fig. 3. The original and their filtered signals in Example 2. 

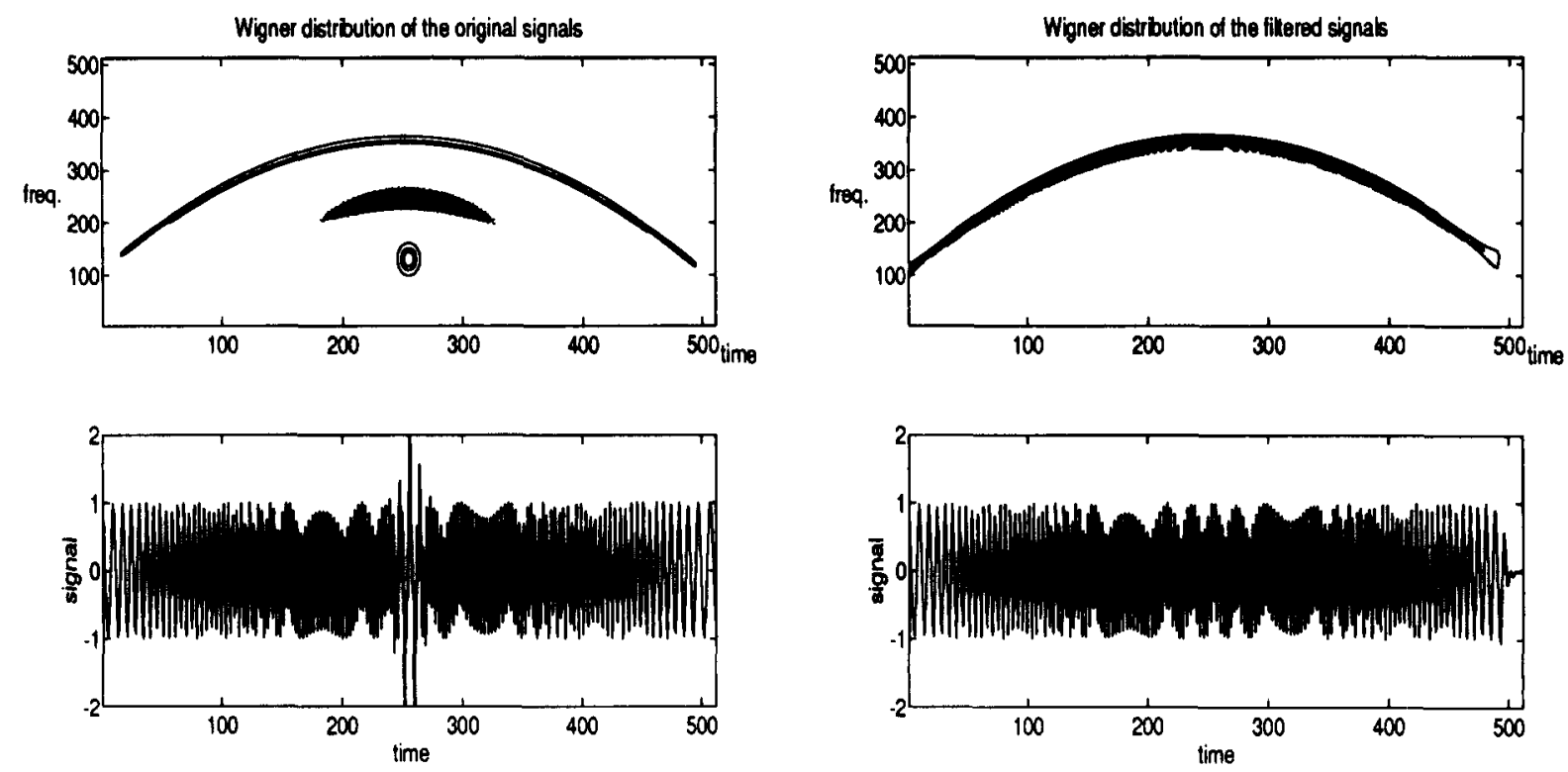

Fig. 4. The original and their filtered signals in Example 3

\section{Experimental results}

In this section, we will make several experiments. Pseudo Wigner distribution is used as a tool to show the results processed by modulation-based time-varying filters. In the following experiments, low-pass FIR filters of length 31 were designed by Park-McClellan algorithm [6].

Example 1. Two chirp signals are separated and not intersected in the time-frequency plane, but overlapped in time and frequency domain, respectively. Fig. 2 shows the Wigner and time domain of original signal and their filtered signals. Our goal is to extract the chirp signal that occurs at time $t=0$. The modulation function in this experiment is a linear FM case. It is a quadratic polynomial of time variable. Its leading coefficient must be positive to modulate the chirp signal to the low-frequency band. There exist some distortions in the filtered signals shown in Fig. 2, and it is due to some transition occurred in the beginning of FIR filtering.

Example 2. Two chirp signals are intersected in the time-frequency plane. Fig. 3 shows the Wigner and time domain of original and their filtered signals in this experiment. One of the chirp's frequencies is increasing, the other is decreasing. Our purpose is to extract the chirp signal whose frequency is increasing. Because two chirps are intersected, they are both passed in the intersection. The modulation function in this example is again a linear FM case. The leading coefficient of polynomial must be positive to get the chirp signal with increasing frequency.

Example 3. A quadratic FM signal and a Gaussian signal are considered in this example. Fig. 4 shows the Wigner and time domain of original and their filtered signals. We want to extract the quadratic FM signal. The modulation function in this experiment is a quadratic FM case. It is a time polynomial of degree 3 . The limitation of this method is the filter bandwidth is fixed, and cannot be varied while processing.

\section{Conclusions}

The modulation-based filters are simple and useful time-variant filters and the Wigner distribution 
is a powerful tool to show the time-frequency distribution of nonstationary signals. We have applied the Wigner distribution as a tool for investigating time-variant filters.

\section{Appendix A}

At first, we will prove Eq. (7). When the LTI filter is treated as LTV filter, 2-D Wigner distribution of time-variant filter can be obtained

$$
\begin{aligned}
& W_{h_{v}}\left(t, t^{\prime}, \omega,-\omega^{\prime}\right) \\
& =\int_{-\infty}^{\infty} \int_{-\infty}^{\infty} h_{v}\left(t+\frac{\tau}{2}, t^{\prime}+\frac{\tau^{\prime}}{2}\right) \\
& \quad \times h_{v}^{*}\left(t-\frac{\tau}{2}, t^{\prime}-\frac{\tau^{\prime}}{2}\right) \mathrm{e}^{-\mathrm{j}\left(\omega \tau-\omega^{\prime} \tau^{\prime}\right)} \mathrm{d} \tau \mathrm{d} \tau^{\prime} \\
& =\int_{-\infty}^{\infty} \int_{-\infty}^{\infty} h_{i}\left(t+\frac{\tau}{2}-t^{\prime}-\frac{\tau^{\prime}}{2}\right) \\
& \quad \times h_{v}^{*}\left(t-\frac{\tau}{2}-t^{\prime}+\frac{\tau^{\prime}}{2}\right) \mathrm{e}^{-\mathrm{j} \omega \tau+\mathrm{j} \omega^{\prime} \tau^{\prime}} \mathrm{d} \tau \mathrm{d} \tau^{\prime} .
\end{aligned}
$$

Let $\lambda=\tau-\tau^{\prime}$,

$$
\begin{gathered}
W_{h_{v}}\left(t, t^{\prime}, \omega,-\omega^{\prime}\right) \\
=\int_{-\infty}^{\infty} h_{i}\left(t-t^{\prime}+\lambda / 2\right) h_{i}^{*}\left(t-t^{\prime}-\lambda / 2\right) \mathrm{e}^{-\mathrm{j} \omega \lambda} \\
\times\left[\int_{-\infty}^{\infty} \mathrm{e}^{-\mathrm{j}\left(\omega-\omega^{\prime}\right) \tau^{\prime}} \mathrm{d} \tau^{\prime}\right] \mathrm{d} \lambda \\
=2 \pi \delta\left(\omega-\omega^{\prime}\right) \int_{-\infty}^{\infty} h_{i}\left(t-t^{\prime}+\frac{\lambda}{2}\right) \\
\times h_{v}^{*}\left(t-t^{\prime}-\frac{\lambda}{2}\right) \mathrm{e}^{-\mathrm{j} \omega \lambda} \mathrm{d} \lambda \\
=2 \pi \delta\left(\omega-\omega^{\prime}\right) W_{h_{i}}\left(t-t^{\prime}, \omega\right) .
\end{gathered}
$$

Eq. (7) has been proved over. Next, we will prove Eq. (10). Let $m(t)$ be the modulation function. $m m\left(t, t^{\prime}\right)=\mathrm{e}^{\mathrm{j}\left[\phi(t)-\phi\left(t^{\prime}\right)\right]}=m(t) m^{*}\left(t^{\prime}\right) . W_{m}(t, \omega)$ is the Wigner distribution of modulation function $m(t)$. $W_{m m}\left(t, t^{\prime}, \omega, \omega^{\prime}\right)$ is the Wigner distribution of $m m\left(t, t^{\prime}\right)$.

Because $h_{v}\left(t, t^{\prime}\right)=h_{i}\left(t-t^{\prime}\right) m m\left(t, t^{\prime}\right)$

$$
\begin{aligned}
& W_{h_{v}}\left(t, t^{\prime}, \omega,-\omega^{\prime}\right) \\
&= {\left[2 \pi \delta\left(\omega-\omega^{\prime}\right) W_{h_{i}}\left(t-t^{\prime}, \omega\right)\right] } \\
& \star_{\omega, \omega^{\prime}} W_{m m}\left(t, t^{\prime}, \omega,-\omega^{\prime}\right) \\
&= 2 \pi\left[\delta\left(\omega-\omega^{\prime}\right) W_{h_{i}}\left(t-t^{\prime}, \omega\right)\right] \\
& \star_{\omega, \omega}\left[W_{m}(t, \omega) W_{m}\left(t^{\prime}, \omega^{\prime}\right)\right] \\
&= 2 \pi \delta\left(\omega-\omega^{\prime}\right) W_{h_{i}}\left(t-t^{\prime}, \omega\right) \\
& \star_{\omega} W_{m}(t, \omega) \star_{\omega^{\prime}} W_{m}\left(t^{\prime}, \omega^{\prime}\right) .
\end{aligned}
$$

The proof of Eq. (10) is completed.

\section{References}

[1] G.F. Boudreaux-Bartels and T.W. Parks, "Time-varying filtering and signal estimation using Wigner distribution synthesis techniques", IEEE Trans. Acoust. Speech Signal Process., Vol. ASSP-34, June 1986, pp. 442-451.

[2] T.A.C.M. Claasen and W.F.G. Mecklenbrauker, "The Wigner distribution - A tool for time-frequency signal analysis, Part I: Continuous-time signals", Philips J. Res., Vol. 35, 1980, pp. 217-250.

[3] N.C. Huang and J.K. Aggarwal, "On linear shift-variant digital filters", IEEE Trans. Circuits Systems, Vol. CAS-27, August 1980 , pp. 672-679.

[4] L. Jacobson and H. Wechsler, "A theory for invariant object recognition in the frontoparallel plane", IEEE Trans. Pattern Anal. Mach. Intel., Vol. PAMI-6, May 1984, pp 325-331.

[5] C.F. Martin, Phase lock Techniques, Wiley, New York, 1979.

[6] A.V. Oppenheim and R.W. Schafer, Discrete-time Signal Processing, Prentice-Hall, Englewood Cliffs, NJ, 1989.

[7] S.C. Pei and T.Y. Wang, "The Wigner distribution of linear time-variant systems", IEEE Trans. Acoust. Speech Signal Process., Vol. ASSP-36, October 1988, pp. 1681-1684. 